\title{
Detecting time-fragmented cache attacks against AES using Performance Monitoring Counters
}

\author{
Iván Prada $^{\dagger}$, Francisco D. Igual and Katzalin Olcoz \\ Departamento de Arquitectura de Computadores y Automática, Universidad \\ Complutense de Madrid, Madrid 28040, Spain \\ ivprada@ucm.es, figual@ucm.es, katzalin@ucm.es
}

\begin{abstract}
Cache timing attacks use shared caches in multi-core processors as side channels to extract information from victim processes. These attacks are particularly dangerous in cloud infrastructures, in which the deployed countermeasures cause collateral effects in terms of performance loss and increase in energy consumption. We propose to monitor the victim process using an independent monitoring (detector) process, that continuously measures selected Performance Monitoring Counters (PMC) to detect the presence of an attack. Ad-hoc countermeasures can be applied only when such a risky situation arises. In our case, the victim process is the Advanced Encryption Standard (AES) encryption algorithm and the attack is performed by means of random encryption requests. We demonstrate that PMCs are a feasible tool to detect the attack and that sampling PMCs at high frequencies is worse than sampling at lower frequencies in terms of detection capabilities, particularly when the attack is fragmented in time to try to be hidden from detection.
\end{abstract}

Keywords: cache attacks, flush+reload, AES, performance monitoring counters

\section{Introduction}

In January 2018, Jann Horn [9] from Google Project Zero and a group of researchers led by Paul Kocher independently disclosed three vulnerabilities, named Spectre (variants 1 and 2) and Meltdown. They discovered that data cache timing could be used to extract information about memory contents using speculative execution. Since that moment, new variants of these transient execution attacks have been disclosed, such as Foreshadow or NetSpectre, to name just two of them [5].

These attacks exploit speculative and out-of-order execution in high performance microarchitectures together with the fact that in modern multi-core architectures some resources are shared across cores. Hence, a malicious process which is being executed in one core of the system can extract information from

\footnotetext{
${ }^{\dagger}$ Corresponding author
} 
a victim executed in a different core. The resource that is most commonly used as side-channel to extract information is the shared cache [2].

This problem is particularly important in cloud environments, where not only multiple users share a multi-core server but also multiple virtual machines can co-reside in the same core due to consolidation in order to save energy. Moreover, the use of simultaneous multithreading techniques, such as Intel's Hyperthreading technology, allow to leverage two or more logical cores per physical core, increasing the degree of resources shared between users.

There has been a proliferation of ad-hoc defenses, mainly microcode and software patches for the operating system and virtual machine monitor. Besides, Intel announced hardware mitigations in its Cascade Lake processors, trying to reduce performance loss due to the countermeasures for some of the attacks [11].

However, the impact of countermeasures on performance is still non negligible, and according to [5] varies from $0 \%$ to almost $75 \%$. Thus, in most situations, security comes at the expense of lower performance and higher energy consumption (due to non-consolidating and disabling hyperthreading).

In this paper, we propose a new attack detection tool that is based on the deployment of a process running in the same core that the victim process it protects, and that detects situations in which an attack is being performed. Following this idea, countermeasures are only taken when the risk level justifies the cost.

The contribution of the paper is two-fold:

- We implement and describe the attack, and design and implement a detector for it based on Performance Monitoring Counters (PMC), evaluating its detection capabilities at different sampling frequencies and showing that high sampling frequencies $(100 \mu \mathrm{s})$ are noisier than lower ones (10 to $100 \mathrm{~ms})$.

- We show that splitting the attack into small pieces and distributing those pieces in time decreases detection capability in a different way for the different detection sampling frequencies. Only low frequencies (bigger than 10 $\mathrm{ms})$ are still able to detect the time-fragmented attack.

The rest of the paper is structured as follows: Section 2 reviews the most relevant works in the field; Section 3 outlines the main concepts needed for the correct understanding of the attack and detection strategy. Then, the attack implemented, detection using PMC and the time-fragmented attack are presented in Sections 4, 5 and 6, respectively. Finally, conclusions are presented in Section 7 .

\section{Related Work}

Detailed surveys on microarchitectural timing attacks in general $[8,2]$ and cache timing attacks in particular [12] can be found in the literature. Besides, Canella [5] performs a systematic evaluation of transient execution attacks. 
Time-driven attacks against the shared and inclusive Last Level Cache (LLC) are mainly based on Flush\&Reload [15] and their variants. So, Briongos [4] extracts the key from the AES T-table based encryption algorithm using improvements over the original attack.

Recently, Performance Monitoring Counters have been used to detect the attack. Chiappeta et al [6] monitor both victim and attacker, while CloudRadar [16] monitors all the virtual machines running in the system. CacheShield [3] only monitors the victim process to detect attacks on both AES and RSA algorithms. None of them considered trying to hide the attack by dividing it into small pieces distributed in time. Our approach is similar to CacheShield [3] in terms of functionality, but we perform a more detailed study of how the specific timing of the attack affects the detection capability.

\section{Background concepts}

For a correct understanding of the attacks and techniques described hereafter, further details on two architectural concepts with direct impact on the attacks are required: cache inclusion policies and memory de-duplication as a specific case of shared memory. Then, the basics of the Flush\&Reload attack are outlined.

\subsection{Shared caches and inclusion policies in modern multi-cores}

Modern multi-core processors feature multi-level caches in which levels can be classified as shared/private across cores and hierarchies as inclusive, noninclusive or exclusive, depending on whether the content of a cache level is present in lower cache levels. Of special interest for us is the combination of shared/inclusive cache levels, such as LLC caches in modern Intel multi-cores; in this scenario, a process executed on a specific core can produce side effects on independent processes executed on a different core. This phenomena can be exploited to perform cache-timing attacks. Supplementary techniques, such as Intel's Cache Allocation Technology (CAT [13]), can be leveraged to isolate specific LLC ways in order to boost performance (reducing contention), but also to mitigate the effects of potential attacks in this type of processors and situations.

\subsection{Shared memory and memory de-duplication}

Modern operating systems, such as Linux, make an intensive use of shared memory across processes to improve memory usage efficiency. Some situations (e.g. parent-child process hierarchies generated through fork()) are easily trackable, but sharing memory pages across independent processes requires ad-hoc sophisticated techniques. This is a very common scenario in multi-virtual machine (multi-VM) deployments sharing the same physical resources, for example.

Memory de-duplication is a specific technique of shared memory, designed to reduce the memory footprint in scenarios in which a hypervisor shares memory pages with the same contents across different virtual machines, but with impact 
also on non-virtualized environments comprising random non-related processes. In the Linux implementation (KSM, Kernel Samepage Merging), a kernel thread periodically checks every page in registered memory sections, and calculates a hash of its contents. This hash is then used to search other pages with identical contents. Upon success, pages are considered identical and merged, saving memory space. Processes that reference to the original pages are updated to point to the merged one. Only after a write operation from one of the VMs (or processes), sharing finishes and the corresponding page is copied by COW (Copy-on-Write).

\subsection{Flush\&Reload}

The FlushERReload attack was first introduced in [15] and it has been used as baseline by later works such as [10], among others. It takes advantage of the combination of inclusive shared caches and memory de-duplication. The basics of the attack are as follows: the attacker runs in a core which shares the last level cache with the victim, and manages to share some page with it through memory de-duplication. It can either contain shared data (i.e. the tables used by the AES encryption algorithm) or shared instructions (for the attack against RSA). In the first phase of the attack (Flush), the attacker evicts the shared blocks from its own private cache, causing the eviction of those data from the shared cache and all the other caches. In the second phase, the victim performs some random work, bringing some of the shared data to the cache again. In the last phase (Reload), the attacker accesses every shared data, measuring the time it takes and it guesses which data have been used by the victim (cache hits) and which ones were not used (cache misses). From this information, the attacker extracts relevant data, such as the AES key.

\section{Implementation of the AES attack}

\subsection{Experimental Setup}

The experimental setup was deployed on a dual-socket server featuring two Intel Xeon Gold 6138 chips with 20-cores each (hyperthreading was disabled), running at 2 Ghz. The memory hierarchy comprises 96 Gbytes DDR4 RAM, 28 Mbytes of unified L3 cache per chip (11-way associative), 1 Mbyte of unified L2 cache per chip (16-way associative) and 32 Kbytes of L1 cache per core (8-way associative). Cache line is 64 bytes. L1 TLB comprises 64 entries (4-way associative) with a page size of 4 Kbytes.

From the software perspective, we employed a Debian GNU/Linux distribution with kernel 4.9.51-1 and GCC 6.3.0. PAPI version 5.5.1.0 [14] built on top of the Linux perf_event subsystem was employed to extract performance counters

information. OpenSSL version 1.1.1.b was used to implement the cryptographic algorithm, compiled with the no-asm flag when using T-tables. 


\subsection{AES algorithm}

The AES algorithm is iterative, so obtaining each encryption needs the execution of several rounds. In $[7,1]$, authors develop the underlying theory of polynomials with coefficients in $G F\left(2^{8}\right)$ (Galois Field of order 256), which is the base for the extraction of transformation values of a single round. The round transformation lies in four steps for the first rounds (SubByte, ShiftRows, MixColumns and AddRoundKey) and three for the last round (all but one, MixColumns). The number of rounds will depend on the length of the key; in our case, for 128bits key, we need 10 rounds. As stated by Daemen and Rijmen in [7], the round transformation of AES can be optimized with 4 look-up tables $\left(T_{i}, i \in 0 \cdots 3\right)$ that contain the pre-calculated values for each of the potential inputs. This way, the encryption round is simplified to a few XOR operations and takes the form:

$$
S_{i, j}=T_{i}\left[s_{i, j}^{k}\right] \oplus \text { RoundKey } y_{i, j}^{k}
$$

for the main rounds, and the last round:

$$
S_{i, j}=T_{(i+2) \% 4}\left[s_{i, j}^{10}\right] \oplus \text { RoundKey } y_{i, j}^{10}
$$

with $S_{i, j}^{k}$ the encrypted char, $s_{i, j}$ the previous state char, $k \in 1 \cdots 9$ the $k$-th round and $s_{1}$ is the original message $\left(s_{0}\right)$ XOR with RoundKey ${ }^{0}$.

\subsection{Implementation of the attack}

The basis of the attack is simple: using T-Tables optimization to extract the last round key (LRK) of AES. In Section 2, we exposed previous algorithms for extraction of the AES key. We use the approach of [4] to break the OpenSSL 1.1.1.b AES 128 bits implementation (this library has had to be compiled with no-asm flag, so that it uses the T-Tables implementation). The attack begins by forcing the de-duplication of library pages (see Section 3.2). This step is mandatory so that victim and attacker can share pages of the dynamic library, hence allowing the observation of memory addresses assigned to AES tables. In order to obtain the origin of the dynamic library, we proceed by opening the library and performing a memory projection (through mmap). Proceeding this way, the KSM daemon will detect a matching in the contents of the mapped file and the loaded dynamic library, and will force the de-duplication. We have experimentally observed a delay of around 300 encryptions to unleash the de-duplication of pages. At that point, the attack can commence. The start addresses of each table are obtained by decompiling the library and determining the offset of each table with respect to its start address.

As seen in Section 2, there are different ways to extract the key based on the information left by the last round of encryption. In this work, we check whether a cache line ${ }^{1}$ resides in L3 upon completion of the encryption process.

\footnotetext{
${ }^{1}$ A cache line -64 bytes in our target architecture- can store 16 elements of a table, provided each element is stored as a 4-byte unsigned integer.
} 
These measurements have been carried out empirically by a Flush\&Reload technique (see Section 3.3) for each one of the four tables. In the following, $T_{j}$ is the corresponding line of the observed table; the attack proceeds by first performing a flush operation of different lines of the table, followed by a random encryption request. The response to this request is then stored $(S[i]$ stores the encrypted text on the $i$-th encryption), together with the information that will be necessary to perform the attack: a matrix $X$ is created and $X_{i j}$ set to 1 if line $T_{j}$ was in L3 after completing the $i$-th encryption, 0 otherwise.

Once these data are obtained, we proceed by searching for the most probable characters belonging to the last round key, following the pseudo-code depicted in Algorithm 1.1, that will return, for each position of the last round key, those characters with the lowest probability. Hence, we will select:

$$
\text { LastRoundKey } y_{i, j}=\min _{t \in 0, \ldots, \text { num_encrypt }} L R K_{i, j}[t]
$$

Once the characters of the last round key have been obtained, the last step is just an inversion of the code used by AES to obtain the last round key, and hence the initial key of the server.

Listing 1.1: Pseudo-code to obtain Last Round Key candidates.

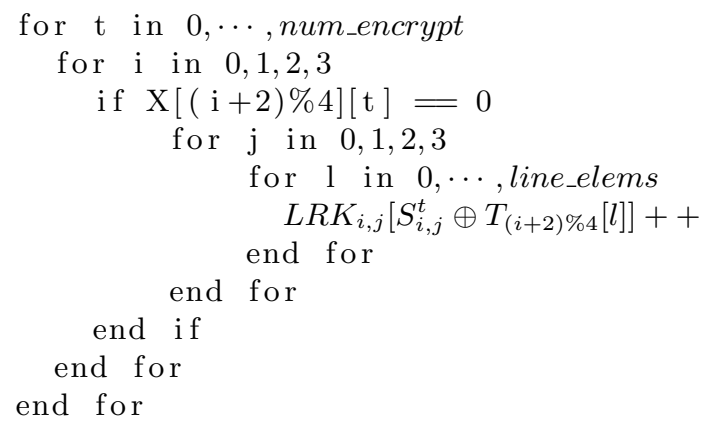

\section{Attack detection using PMCs}

Cache timing attacks cause an anomalously high number of L3 misses, due to the flush and reload activity; hence, measuring L3 misses is an straightforward mechanism to detect them. As explained in Section 2, there have been some works in this field and most of them use L3 misses.

In addition to L3 cache misses, we chose the total number of load instructions executed by the victim as a way to estimate the number of encryptions being performed by the victim, so that the ratio between both counters provides a metric that is constant for different levels of load in the victim. Thus, our detection metric is the amount of L3 cache misses (in thousands) per load instruction (1000*L3 misses/LD instruction). 


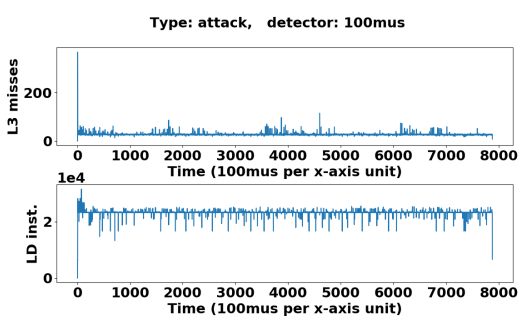

(a) Attack. Detection freq.: $100 \mu \mathrm{s}$ Type: attack, detector: $1 \mathrm{~ms}$
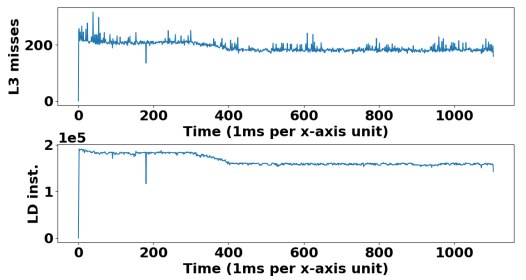

(c) Attack. Detection freq.: $1 \mathrm{~ms}$ Type: attack, detector: 10ms
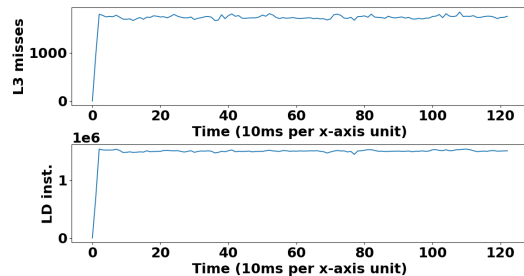

(e) Attack. Detection freq.: $10 \mathrm{~ms}$ Type: attack, detector: 100ms
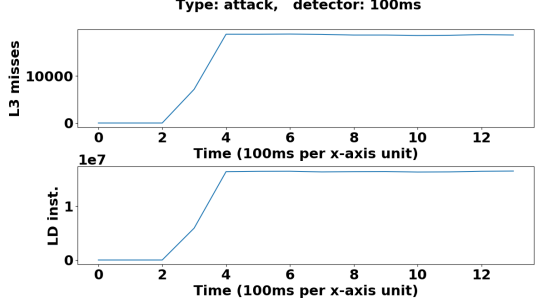

(g) Attack. Detection freq.: $100 \mathrm{~ms}$

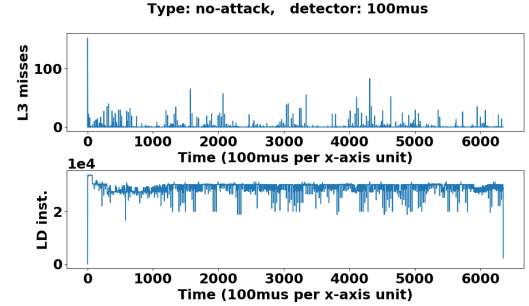

(b) No attack. Detection freq.: $100 \mu \mathrm{s}$
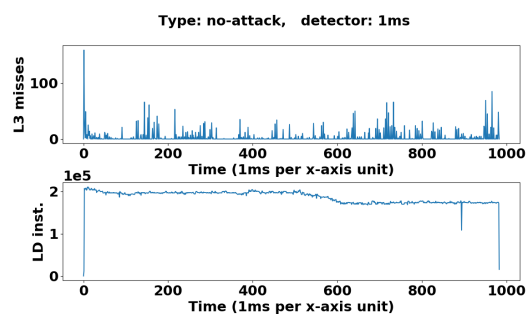

(d) No attack. Detection freq.: $1 \mathrm{~ms}$ Type: no-attack, detector: $10 \mathrm{~ms}$
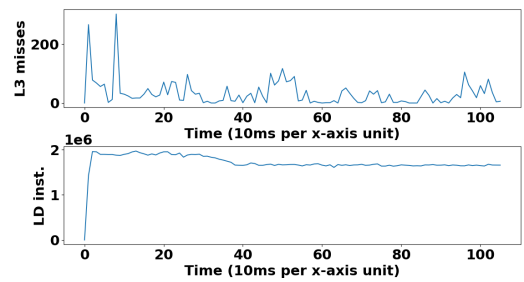

(f) No attack. Detection freq.: $10 \mathrm{~ms}$
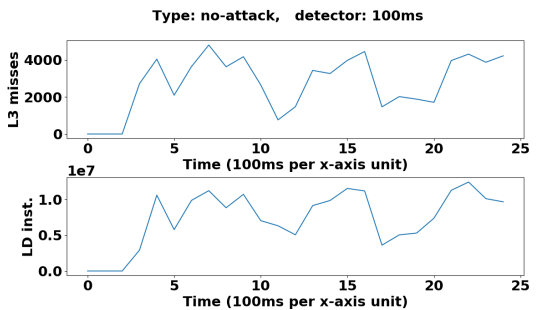

(h) No attack. Detection freq.: $100 \mathrm{~ms}$

Fig. 1: Results obtained from performance counters at different sampling rates. Each one of the four rows reports the results obtained for the L3 cache misses (above) and number of load instructions (below) in the victim under attack (left) and with no attack (right). The rows correspond to the different sampling rates analyzed: $100 \mu \mathrm{s}$ (first row), $1 \mathrm{~ms}$ (second row), $10 \mathrm{~ms}$ (third row) and $100 \mathrm{~ms}$ (last row). 
Figure 1 reports the values of the chosen PMCs for the victim both in the presence and absence of attack. The experiment was repeated at different sampling frequencies, to study the effect of the sampling frequency in the detection capability. Figure 2 shows the values of the proposed metric for the results in Figure 1. The first observation is that the selected metric is an effective mechanism to detect the attack; the values under attack are close to 1 while the values without attack are 10 to 100 times lower. In this situation, the attack is detected if, after the initial cold misses (identified as $100 \mathrm{~ms}$ in our experiments), the value remains close to 1 .

A second conclusion from Figure 2 is that sampling PMCs at $100 \mu \mathrm{s}$ leads to more noisy results for the no-attack experiment. Given that this sampling rate also produces a higher overhead, we will not use that sampling frequency in the following.

\section{Analysis of a time-fragmented attack}

In this section, we propose a complete set of experiments in order to determine if the division of the attack in discrete pieces and their distributed execution in time can potentially disguise the attack and invalidate the action of our detector.

We proceed by dividing the 50,000 encryptions needed for the attack into equally-sized groups (or "packets" in the following) of encryptions. We have evaluated packets of decreasing sizes, namely: 5, 000, 500, 50 and 5 encryptions. Furthermore, in order to analyze the effect of increasing the gap (time) between packets, we call "interval" to the separation between two consecutive packets. In our experiments, we vary the interval between packets from $10 \mu$ s to $100 \mathrm{~ms}$. For each combination of packet size and interval, we used the sampling rates of the previous section except the highest one: $1 \mathrm{~ms}, 10 \mathrm{~ms}$ and $100 \mathrm{~ms}$.

The most interesting results are obtained for small packets and large intervals, as expected. Figure 3 shows the results when the attack is divided into 100 packets of 500 encryptions, and the time interval between two consecutive packets is $10 \mathrm{~ms}$. The sampling rate is either $1 \mathrm{~ms}$ or $10 \mathrm{~ms}$. The metrics obtained from the $10 \mathrm{~ms}$ samples are close to the usual value for the attack, but the results for $1 \mathrm{~ms}$ samples switch from the values corresponding to an attack (close to 1) to the no-attack values (close to 0). As expected, for the high resolution frequency, some samples do not find any difference between attack and no-attack, because they fall in the interval of time between packets of the attack. On the contrary, the low resolution samples always find the "big picture".

Figure 4 reports an equivalent evaluation for packets 10 times smaller, with the aim of reducing the time in which the attack can be detected. In this case, the difference between the obtained results at different sampling rates is more evident. The time interval between two consecutive packets is $10 \mathrm{~ms}$ and the sampling rates are 1, 10 and $100 \mathrm{~ms}$. For the $1 \mathrm{~ms}$ sampling rate, on one hand, the no-attack experiment has higher number of L3 misses due to the separation between packets. During those intervals some cache lines are evicted due to normal functioning of the system. On the other hand, the experiment with attack 


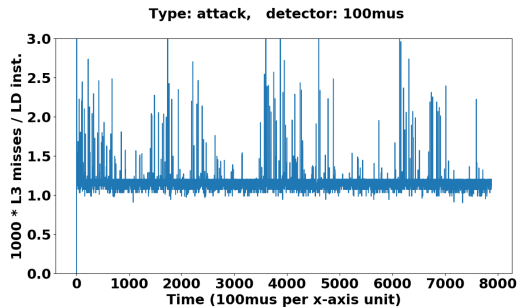

(a) Attack. Detection freq.: $100 \mu \mathrm{s}$

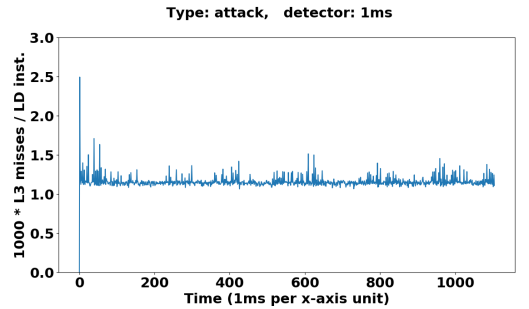

(c) Attack. Detection freq.: $1 \mathrm{~ms}$ Type: attack, detector: $10 \mathrm{~ms}$

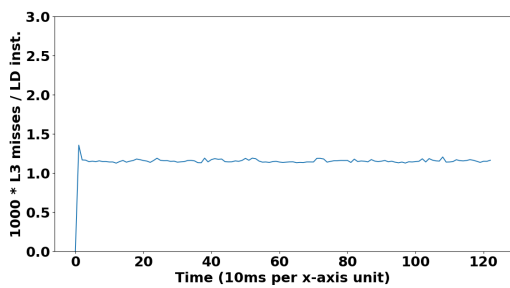

(e) Attack. Detection freq.: $10 \mathrm{~ms}$

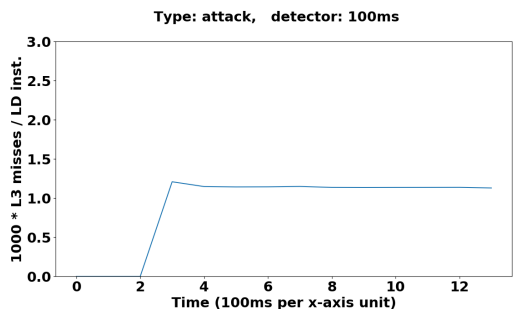

(g) Attack. Detection freq.: $100 \mathrm{~ms}$

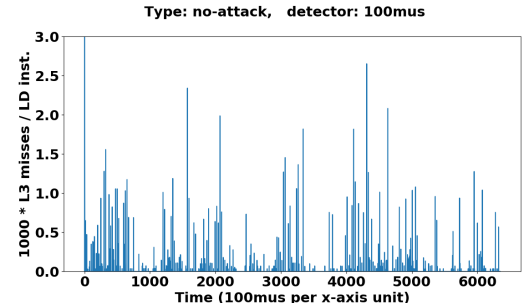

(b) No attack. Detection freq.: $100 \mu \mathrm{s}$

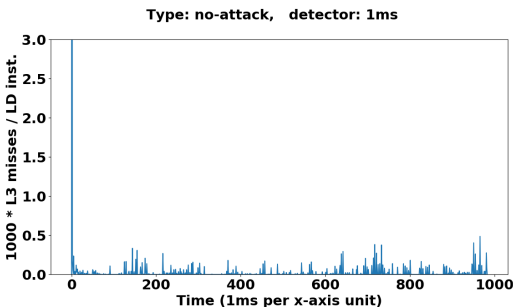

(d) No attack. Detection freq.: $1 \mathrm{~ms}$

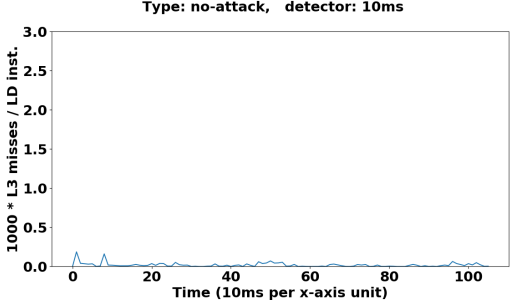

(f) No attack. Detection freq.: $10 \mathrm{~ms}$

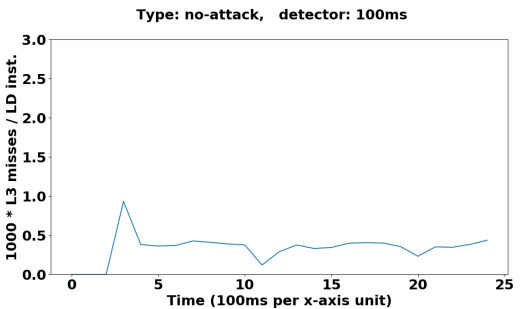

(h) No attack. Detection freq.: $100 \mathrm{~ms}$

Fig. 2: Metric evaluation for attack detection at different sampling rates. Each row displays the proposed metric: 1000 L3 cache misses per load instruction in the victim under attack (left-side column) and without attack (right-side column). The four rows correspond to the four sampling rates analyzed: $100 \mu \mathrm{s}$ (first row), $1 \mathrm{~ms}$ (second row), $10 \mathrm{~ms}$ (third row) and $100 \mathrm{~ms}$ (last row). 
also switches from low to high values of the metric as in the previous experiment. This fact can be observed in Figure 5, which is an augmented view of the results for the attack with $1 \mathrm{~ms}$ sampling. It confirms that the attack can be more easily hidden from the high resolution samples than from the lower ones.

Finally, the packet size is decreased to 5 encryptions. In this experiment, when the interval between packets is longer than $1 \mathrm{~ms}$ the attack stops working. The results for $1 \mathrm{~ms}$ interval are show in Figure 6 and they confirm that our detection metric is able to detect the attack with a sampling rate of $10 \mathrm{~ms}$ or bigger.

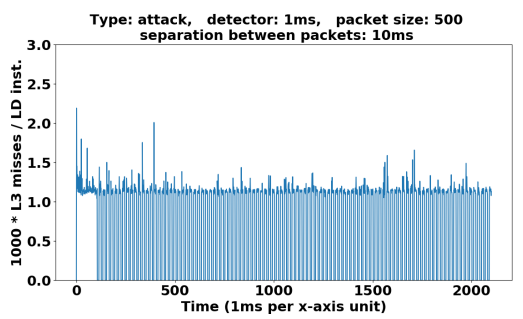

(a)

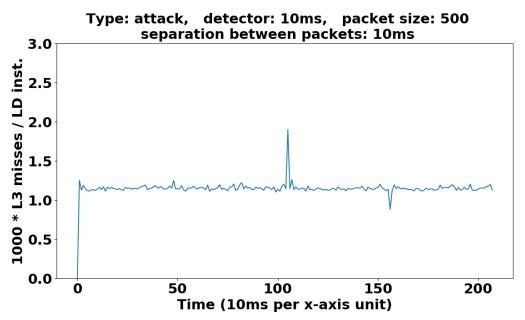

(c)

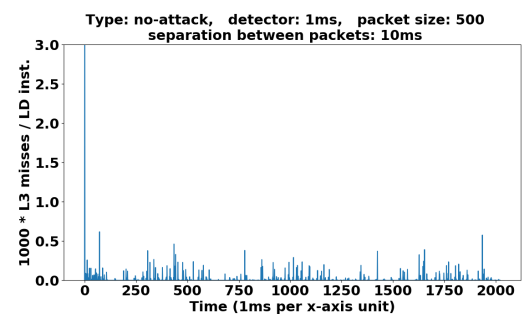

(b)

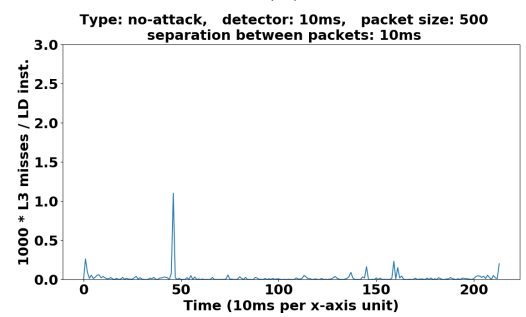

(d)

Fig. 3: Detection metric for packets of 500 encryptions with interval of $10 \mathrm{~ms}$. There is an attack in the left column and no attack in the right one. The sampling rate is $1 \mathrm{~ms}$ (top) and $10 \mathrm{~ms}$ (bottom).

\section{Conclusions}

In this paper, we proposed a mechanism to protect victim processes running in multi-core servers (either native or inside a VM) against cache timing attacks by adding to the server a new detector process that monitors only the PMCs associated to the victim process. To that end, we implemented a cache timing attack against the table based AES encryption algorithm. We used 1000 L3 cache misses per load instruction as a detection metric and achieved detection of the attack for all the different sampling rates, although sampling at high frequency is worse than at lower ones. 


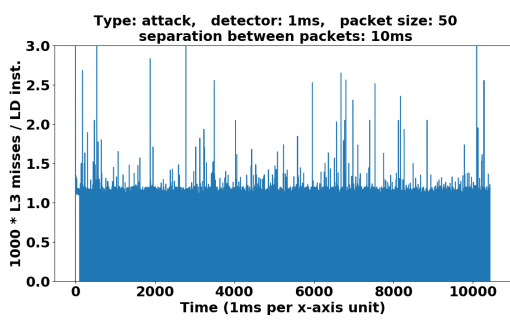

(a)

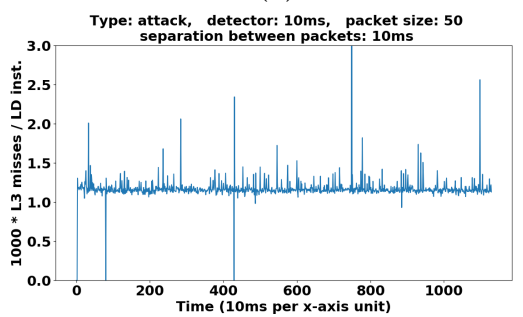

(c)

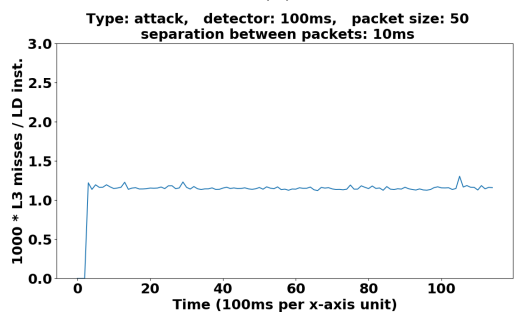

(e)

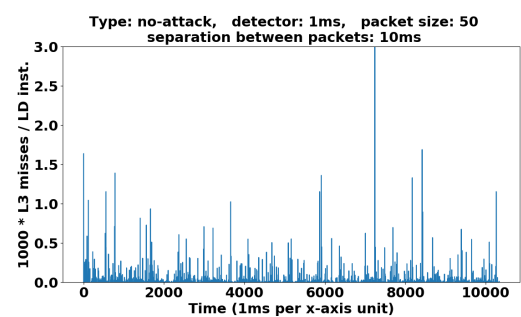

(b)

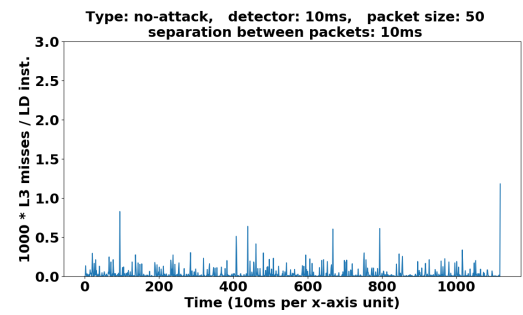

(d)

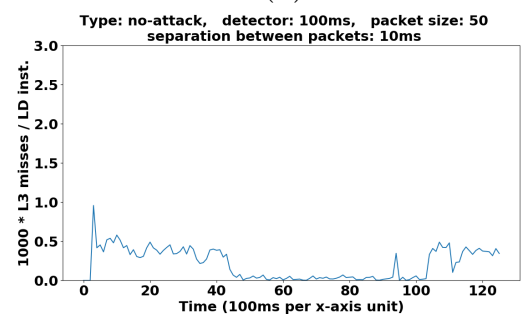

(f)

Fig. 4: Detection metric for packets of 50 encryptions with interval of $10 \mathrm{~ms}$. There is an attack in the left column and no attack in the right one. The sampling rate is $1 \mathrm{~ms}$ (top), $10 \mathrm{~ms}$ (middle), and $100 \mathrm{~ms}$ (bottom).

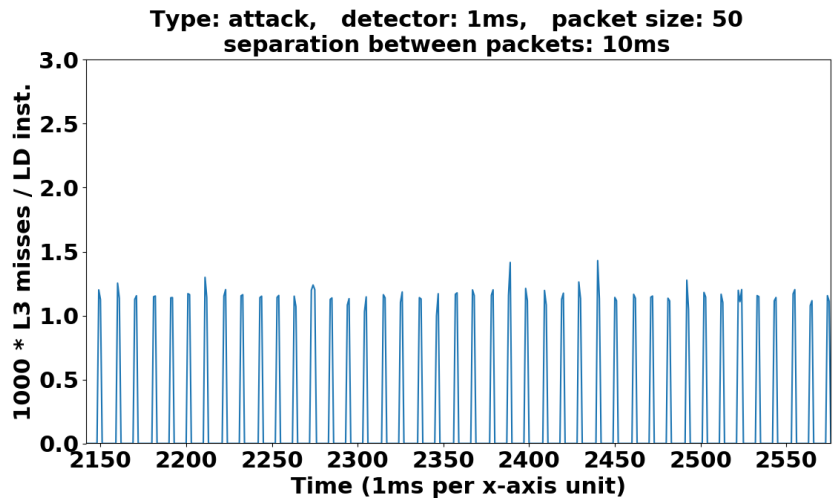

Fig. 5: Augmented view of the detection metric for the attack with packets of 50 encryptions, interval of $10 \mathrm{~ms}$ and sampling rate of $1 \mathrm{~ms}$. 


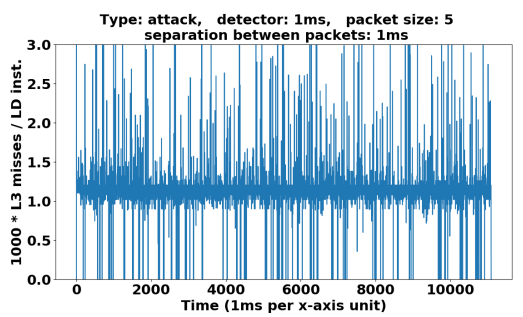

(a)

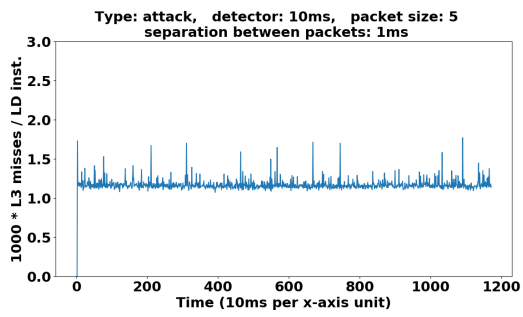

(c)

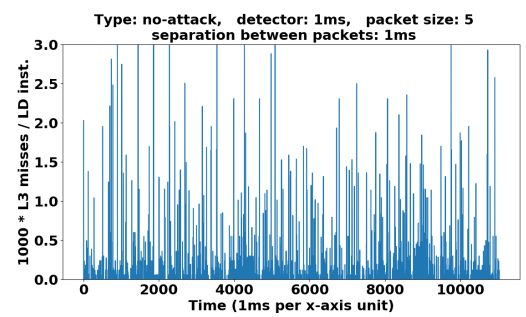

(b)

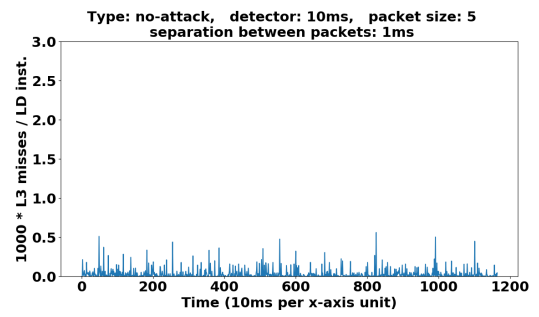

(d)

Fig. 6: Detection metric for packets of 5 encryptions with interval of $1 \mathrm{~ms}$. There is an attack in the left column and no attack in the right one. The sampling rate is $1 \mathrm{~ms}$ (above) and $10 \mathrm{~ms}$ (below).

We have tried to hide the attack dividing it into small parts and interleaving time slots with attack and without attack. Thus, sampling PMC at high frequency makes detection of the attack more difficult. Again, lower frequency monitoring (10 ms and $100 \mathrm{~ms}$ ) results in higher detection capability.

\section{Acknowledgements}

This work is supported by the EU FEDER and the Spanish MINECO under grant number TIN2015-65277-R and by Spanish CM under grant S2018/TCS4423. We would like to thank Samira Briongos and Pedro Malagón for their helpful comments on some details of the attack implementation.

\section{References}

1. Specification for the advanced encryption standard (aes). Federal Information Processing Standards Publication 197 (2001). URL http://csrc.nist.gov/publications/ fips/fips197/fips-197.pdf

2. Biswas, A.K., Ghosal, D., Nagaraja, S.: A Survey of Timing Channels and Countermeasures. ACM Computing Surveys 50(1), 1-39 (2017). DOI 10.1145/3023872

3. Briongos, S., Irazoqui, G., Malagón, P., Eisenbarth, T.: CacheShield: Detecting Cache Attacks through Self-Observation. Codaspy pp. 224-235 (2018). DOI $10.1145 / 3176258.3176320$ 
4. Briongos, S., Malagón, P., de Goyeneche, J.M., Moya, J.: Cache Misses and the Recovery of the Full AES 256 Key. Applied Sciences 9(5), 944 (2019). DOI 10.3390/app9050944

5. Canella, C., Van Bulck, J., Schwarz, M., Lipp, M., von Berg, B., Ortner, P., Piessens, F., Evtyushkin, D., Gruss, D.: A Systematic Evaluation of Transient Execution Attacks and Defenses (2018). URL http://arxiv.org/abs/1811.05441

6. Chiappetta, M., Savas, E., Yilmaz, C.: Real time detection of cache-based sidechannel attacks using hardware performance counters. Applied Soft Computing Journal 49, 1162-1174 (2016). DOI 10.1016/j.asoc.2016.09.014

7. Daemen, J., Rijmen, V.: The design of Rijndael: AES - the Advanced Encryption Standard. Springer-Verlag (2002)

8. Ge, Q., Yarom, Y., Cock, D., Heiser, G.: A survey of microarchitectural timing attacks and countermeasures on contemporary hardware. Journal of Cryptographic Engineering 8(1), 1-37 (2018). DOI 10.1007/s13389-016-0141-6

9. Horn, J.: Project zero - Reading privileged memory with a sidechannel (2018). URL https://googleprojectzero.blogspot.com/2018/01/ reading-privileged-memory-with-side.html

10. Irazoqui, G., Inci, M., Eisenbarth, T., Sunar, B.: Wait a minute! A fast, cross-VM attack on AES. In: Lecture Notes in Computer Science, vol. 8688 LNCS, pp. 299319 (2014). DOI 10.1007/978-3-319-11379-1_15. URL http://link.springer.com/ 10.1007/978-3-319-11379-1_15

11. Kumar, A., Kottapalli, S., Steiner, I.M., Valentine, B., Hirsh, I., Vedaraman, G., Looi, L.P., Arafa, M., Rudoff, A., Mandava, S., Fahim, B., Vora, S.A.: Future Intel Xeon Scalable Processors. Hotchips (2018)

12. Lyu, Y., Mishra, P.: A Survey of Side-Channel Attacks on Caches and Countermeasures. Journal of Hardware and Systems Security 2(1), 33-50 (2017). DOI 10.1007/s41635-017-0025-y

13. Nguyen, K.T.: Introduction to Cache Allocation Technology in the Intel@ Xeon® Processor E5 v4 Family (2016). URL https://software.intel.com/en-us/articles/ introduction-to-cache-allocation-technology

14. Terpstra, D., Jagode, H., You, H., Dongarra, J.: Collecting performance data with PAPI-C. In: Proceedings of the 3rd International Workshop on Parallel Tools for High Performance Computing 2009, pp. 157-173. Berlin, Heidelberg (2010). DOI 10.1007/978-3-642-11261-4-11

15. Yarom, Y., Falkner, K.: Flush+reload: A high resolution, low noise, 13 cache sidechannel attack. In: Proceedings of the 23rd USENIX Conference on Security Symposium, pp. 719-732 (2014)

16. Zhang, T., Zhang, Y., Lee, R.B.: CloudRadar: A Real-Time Side-Channel Attack Detection System in Clouds. In: Proceedings of the International Symposium on Research in Attacks, Intrusions and Defenses (2016). DOI 10.1007/ 978-3-319-45719-2_6 